\title{
Efecto de diferentes condiciones de almacenamiento sobre el comportamiento poscosecha del cebollín (Allium schoenoprasum L.)
}

\section{Effect of different storage conditions on the postharvest behavior of chive plants (Allium schoenoprasum L.)}
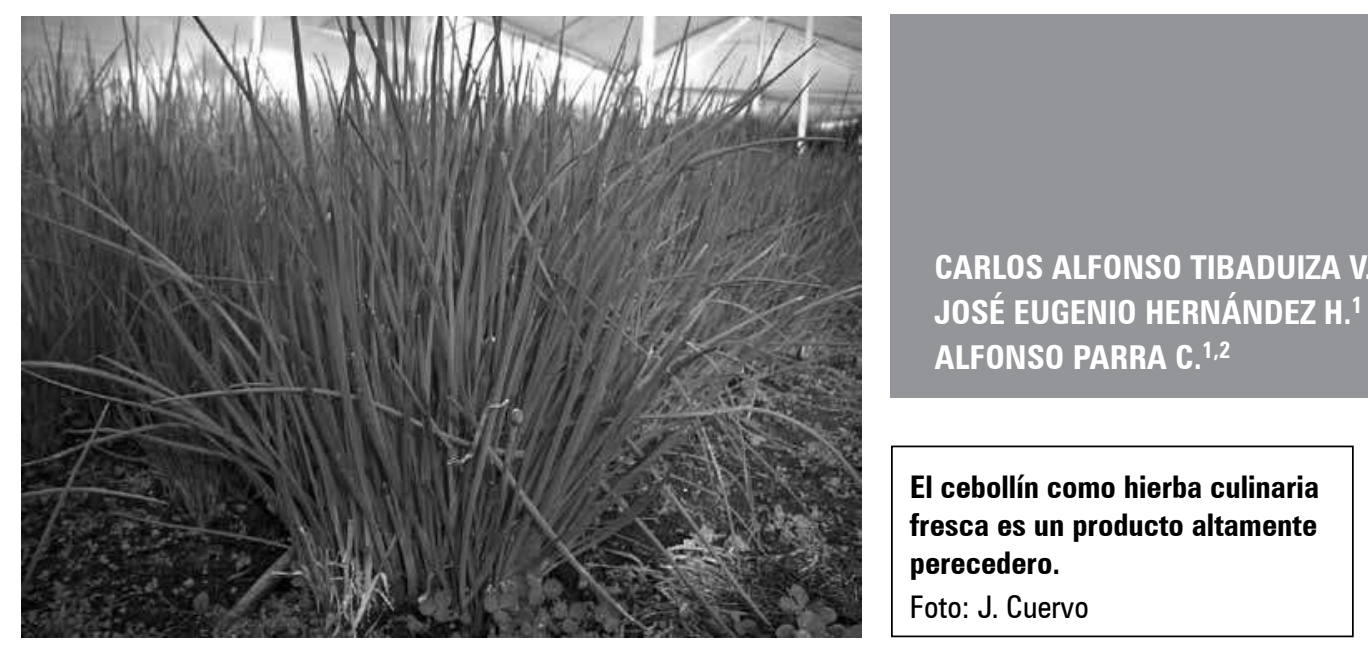

\section{RESUMEN}

Hojas de cebollín fresco se empacaron en bolsas de polietileno de baja densidad de $500 \mathrm{~g}$ de capacidad y se dispusieron en cajas de cartón, para caracterizar el comportamiento fisiológico del conjunto producto-empaqueembalaje almacenado a $3^{\circ} \mathrm{C}, 80 \%$ humedad relativa (HR) y $18^{\circ} \mathrm{C}, 60 \% \mathrm{HR}$ (que son las condiciones de manejo actual por parte de la mayoría de los exportadores) y evaluar dos porcentajes de perforación (1,00 y 1,38\%) del empaque de polietileno de baja densidad y del embalaje de cartón a $3^{\circ} \mathrm{C}$ y $80 \% \mathrm{HR}$. El conjunto cebollínempaque-embalaje se caracterizó fisiológicamente a través de su intensidad respiratoria, variación de peso y contenido de humedad físicamente midiendo los cambios presentados en peso, longitud y volumen. A $3^{\circ} \mathrm{C}$ el producto mantuvo condiciones deseables de comercialización por 11 días durante 5 días a $18^{\circ} \mathrm{C}$. Los valores de intensidad respiratoria promedio fueron de 75 y $260 \mathrm{mg} \mathrm{CO}_{2} \mathrm{~kg}^{-1} \mathrm{~h}^{-1}$, respectivamente. El embalaje con $0 \%$ de perforación aisló el cebollín de la humedad relativa de almacenamiento y mantuvo el contenido de humedad del inicio del almacenamiento, en un $88 \%$. Las pérdidas de peso a lo largo del experimento fueron menores al $4 \%$. Al final del almacenamiento, en cada condición, las hojas de cebollín presentaron amarillamiento y olor desagradable. La investigación establece que el cambio de empaque bajo las condiciones actuales de manejo no es relevante; las películas de polietileno son efectivas y se recomienda sin embargo diámetros de perforación menores a $4 \mathrm{~mm}$ y buena uniformidad en su distribución.

1 Facultad de Ingeniería, Departamento de Ingeniería Civil y Agrícola, Universidad Nacional de Colombia, Bogotá (Colombia).

2 Autor para correspondencia.aparrac@unal.edu.co 


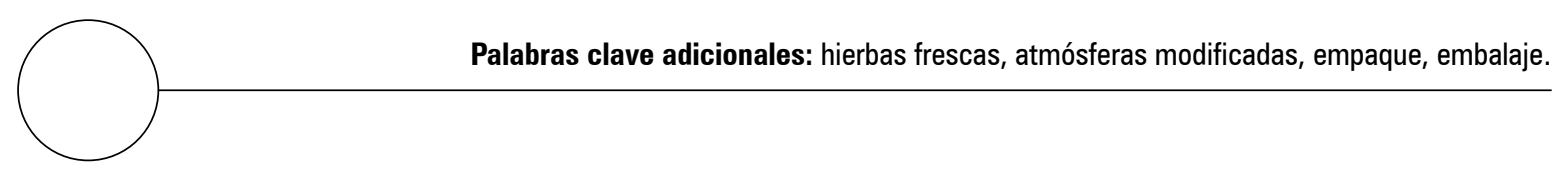

\section{ABSTRACT}

Leaves of fresh chives were packed in low density $500 \mathrm{~g}$ polyethylene bags and placed in cardboard boxes to characterize the physiological behavior of product-packing storage at $3^{\circ} \mathrm{C}, 80 \%$ relative humidity $(\mathrm{RH})$ and $18^{\circ} \mathrm{C}, 60 \% \mathrm{RH}$ (the current handling conditions by most exporters) and evaluate two perforation rates (1.00 and $1.38 \%$ ) of low density polyethylene film, and 0 to $9 \%$ cardboard packaging storage at $3{ }^{\circ} \mathrm{C}$ and $80 \% \mathrm{RH}$. The chives-bag-packaging combinations were characterized physiologically by respiratory rate, variation in weight and moisture content, and changes of weight, length and volume were physically measured. At $3^{\circ} \mathrm{C}$, the product remained in desirable marketing conditions for 11 days and for 5 days at $18^{\circ} \mathrm{C}$; the average respiratory rate values were 75 and $260 \mathrm{mg} \mathrm{CO}_{2} \mathrm{~kg}^{-1} \mathrm{~h}^{-1}$, respectively. The chives in packages with $0 \%$ perforation were insulated from the relative humidity of the storage area and maintained the initial storage moisture content at $88 \%$. Weight loss during the experiment was less than $4 \%$. At the end of storage, for each condition, the chives showed yellowing leaves and had an unpleasant odor. The investigation established that a change of packaging under current management conditions is not relevant; polyethylene films are effective and recommended with perforations with diameters of less than $4 \mathrm{~mm}$ and a uniform distribution.

Additional key words: fresh herbs, modified atmosphere, package, boxing.

El cebollín (Allium schoenoprasum L.) es una hierba culinaria muy apetecida por sus características organolépticas, perteneciente a la familia Alliaceae. La planta posee un hábito de crecimiento perenne y presenta muchos ciclos de autorrenovación de sus estructuras vegetativas a través de los bulbillos (Delahaut y Newenhouse, 2003; Abello et al., 2006). El órgano de interés económico del cebollín está representado por sus hojas, las cuales son cilíndricas, tubulares y delgadas, con una longitud superior a $17 \mathrm{~cm}$, poseen un delicado aroma que las hace ser preferidas por los gourmet para destacar el sabor de diferentes pla- tos, su consumo es generalmente en fresco (Bernal et al., 2008). El cebollín se produce en climas fríos de Suramérica tropical, entre 2.000 y 2.800 msnm y bajo invernadero, aunque también se puede establecer a libre exposición, adaptándose a diferentes tipos de suelo (Barreño, 2006).

La planta de cebollín es cultivada y consumida especialmente en el hemisferio norte, aunque en la actualidad su cultivo y comercialización se extiende por todo el mundo. La producción de hierbas frescas en Colombia se ha desarrollado debido a su rusticidad relativa para adaptarse a 
las condiciones ambientales en donde se cultiva, la producción no estacional y a la calidad competitiva del producto fresco en el mercado internacional (Barreño, 2005).

La ubicación geográfica en el trópico y su producción durante todo el año le han permitido a esta especie cobrar gran fuerza en las exportaciones colombianas con una participación del 8\% con respecto a las demás hierbas aromáticas culinarias vendidas en fresco (Bernal et al., 2008). Los países principales a los cuales se exporta cebollín son Estados Unidos, Canadá, Inglaterra, Alemania y Holanda (Barreño y Clavijo, 2006).

La calidad del cebollín es la característica más importante para su comercialización. Los parámetros de calidad son en su mayor parte visuales e incluyen: hojas turgentes (apariencia fresca), uniformidad de tamaño, bien formados (como máximo levemente curvados), color brillante (verde intenso), bien limpios, y libres de raíces, pudriciones, daño de insectos, daño mecánico, hojas quebradas o rotas, o puntas cortadas deshidratadas (Ángel, 2004).

El cebollín como hierba culinaria fresca es un producto altamente perecedero, sus hojas como parte comestible son muy delicadas y pierden la frescura rápidamente, lo cual genera que el tiempo de almacenamiento sea reducido. La senescencia de esta planta está indicada por pérdida de clorofila y amarillamiento de las hojas con su posterior deterioro (Imahori et al., 2007). Durante la senescencia del cebollín se presenta degradación de proteínas con liberación y acumulación de aminoácidos, degradación de clorofilas y muchos otros componentes tales como ácido ascórbico y lípidos, que son esenciales para la calidad sensorial y nutricional de esta especie (Imahori et al., 2004). Para el cebollín se ha identificado que la tasa de senescencia depende de varios factores incluyendo el cultivar y la madurez de las hojas en la cosecha, temperatura de almacenamiento, composición de la atmósfera y duración del almacenamiento (Imahori et al., 2007).

Las mejores condiciones para la conservación de la mayoría de las hierbas frescas (a excepción de la albahaca) se obtienen a temperaturas cercanas a los $0^{\circ} \mathrm{C}$ (Cantwell y Reid, 1993; Ángel, 2004; Sanabria, 2004), y se mejora notablemente si adicionalmente se hace un correcto manejo de la modificación gaseosa (niveles adecuados de $\mathrm{CO}_{2}, \mathrm{O}_{2}$ y $\mathrm{N}_{2}$ ) de la atmosfera de almacenamiento (Parra, 2007). Las hierbas frescas por lo general se comercializan empacadas, siendo el material de la película de empaque muy importante para que provea tasas de transmisión y concentraciones adecuadas de los gases $\left(\mathrm{CO}_{2}, \mathrm{O}_{2}\right.$, $\mathrm{N}_{2}$, y vapor de agua), proporcionando atmósferas modificadas (AM) que favorezcan la conservación de estos productos frescos.

La AM tiene como objetivo principal cambiar el microambiente del producto almacenado y debe considerarse como una técnica complementaria a un adecuado manejo de la temperatura y humedad relativa de almacenamiento del producto (Parra, 2007). La técnica de AM es utilizada en la prolongación de la vida de almacenamiento de productos frescos; los efectos fisiológicos benéficos de esta técnica son la reducción de la tasa respiratoria, disminución de la acción y biosíntesis del etileno, control de algunos desórdenes fisiológicos como el daño por frío en los tejidos vegetales y un retraso de la senescencia, esto directamente mediado por presiones parciales bajas en $\mathrm{O}_{2}$ y/o altas en $\mathrm{CO}_{2}$ (Yahia, 2009; Parra, 2007). El uso de esta técnica ha sido reportada en albahaca (Camargo, 2008), lechuga (Smyth et al., 1998), cebollín chino (Imahori et al., 2004; 2007).

Los valores promedio de intensidad respiratoria para cebollín reportados por Sanabria (2004) para una réplica de tres unidades de $100 \mathrm{~g}$ cada uno, oscilan entre $117,39 \mathrm{mg} \mathrm{CO} \mathrm{kg}^{-1} \mathrm{~h}^{-1}$ para producto empacado en bolsa de polietileno y almacenado a $3^{\circ} \mathrm{C}$ y de $139,35 \mathrm{mg} \mathrm{CO} \mathrm{kg}^{-1} \mathrm{~h}^{-1}$ 
para producto almacenado a $18^{\circ} \mathrm{C}$ el primer día. En el día 3 de almacenamiento el cebollín reduce su intensidad respiratoria en las dos condiciones (3 y $18^{\circ} \mathrm{C}$ ) a valores de 58,64 y 90,49 $\mathrm{mg} \mathrm{CO}_{2}$ $\mathrm{kg}^{-1} \mathrm{~h}^{-1}$ respectivamente. Este estudio también reporta que el producto se mantuvo durante 5 d almacenado a $18^{\circ} \mathrm{C}$. Ángel (2004) realizó estudios del comportamiento del cebollín empacado en bolsas de polietileno y encontró que la intensidad respiratoria osciló entre 25 y $39 \mathrm{mg} \mathrm{CO}_{2} \mathrm{~kg}^{-1}$ $\mathrm{h}^{-1}$. El peso de las unidades evaluadas fue de 100 g. Sin embargo, los datos y las curvas de intensidad respiratoria que son presentados en estos dos trabajos no siguen una tendencia marcada sino que tienen un comportamiento aleatorio, y los autores no dan una explicación clara de este comportamiento, el cual puede deberse posiblemente a errores experimentales.

Medina (1988), determinó la tendencia respiratoria de la cebolla junca (Allium fistulosum) almacenada a $11^{\circ} \mathrm{C}$ y $75 \%$ de $\mathrm{HR}$, y encontró que su comportamiento es el de un producto no climatérico, con una intensidad respiratoria variando de $255 \mathrm{mg} \mathrm{CO}_{2} \mathrm{~kg}^{-1} \mathrm{~h}^{-1}$ al inicio del almacenamiento, hasta un valor de $180 \mathrm{mg} \mathrm{CO}_{2} \mathrm{~kg}^{-1} \mathrm{~h}^{-1}$ en el día 5 de almacenamiento, cuando perdió su calidad comercial.

Con el fin de caracterizar el efecto de diferentes condiciones de almacenamiento sobre el comportamiento fisiológico y la calidad poscosecha del cebollín, en la presente investigación se evaluaron dos condiciones de almacenamiento, en las cuales se consideró la implicación e importancia del empaque y el embalaje sobre el comportamiento fisiológico durante el almacenamiento refrigerado.

\section{MATERIALES Y MÉTODOS}

\section{Material vegetal}

El cebollín fue obtenido de las empresas de exportación Sagra y Aromáticas de la Sabana, ubi- cadas en los municipios de Chía (2.500 msnm) y Guasca (2.600 msnm) en el departamento de Cundinamarca (Colombia) respectivamente, en donde creció bajo condiciones comerciales. Siguió un proceso de preenfriamiento en cuarto refrigerado a $2^{\circ} \mathrm{C}$ durante $2 \mathrm{~h}$, luego fue empacado en bolsas de polietileno de baja densidad y estas a su vez se dispusieron en cajas telescópicas en 12 unidades; y posteriormente se llevaron al laboratorio de frutas y hortalizas del Departamento de Ingeniería Civil y Agrícola de la Facultad de Ingeniería de la Universidad Nacional de Colombia, sede Bogotá, para su respectivo estudio.

Diseño experimental y tratamientos. Los experimentos fueron organizados en un diseño completamente aleatorio en la primera etapa, en la cual se evaluó el producto a temperaturas de 3 y $18^{\circ} \mathrm{C}$, bajo el mismo empaque y disposición del producto en el embalaje. Para la segunda, en un diseño factorial de segundo grado, los cuatro tratamientos establecidos se dispusieron a $3^{\circ} \mathrm{C}$ y $80 \%$ de humedad relativa (HR), fueron (1) Empaque actual, caja actual (EaCa); (2) empaque actual, caja nueva ( $\mathrm{EaCn})$; (3) empaque nuevo, caja actual (EnCa) y (4) empaque nuevo, Caja nueva (EnCn).

El empaque nuevo propuesto fue la bolsa de polietileno de baja densidad con un nivel de perforación de 1,38\% (huecos de $4 \mathrm{~mm}$ ), superior al actual que es de $1 \%$ (huecos de $6 \mathrm{~mm}$ ), y el embalaje propuesto fue una caja de cartón con perforación de $9 \%$, para compararla con la actual de $0 \%$ de perforación (telescópica).

Durante la investigación se evaluó la disposición actual de exportación del conjunto cebollínempaque-embalaje y se dividió en dos etapas; en la primera se caracterizó el comportamiento fisiológico del cebollín bajo dos condiciones de almacenamiento $\left(3^{\circ} \mathrm{C}, 80 \% \mathrm{HR}\right.$ y $18^{\circ} \mathrm{C}, 60 \%$ HR), y en la segunda etapa se definió a partir de los resultados, recomendaciones y observaciones técnicas establecidas en la primera, el manejo del producto a baja temperatura $\left(>0^{\circ} \mathrm{C}\right)$ y la elección 
de un material de empaque con permeabilidad adecuada.

\section{Caracterización física}

Se realizó siguiendo el procedimiento utilizado por Sanabria (2004): se contó el total de las unidades (tallos) de la muestra (50 g), se determinó el peso individual de cada tallo (g), mediante una balanza electrónica marca Mettler PC2000 (precisión 0,01 g), también se determinaron las dimensiones longitud $(\mathrm{cm})$ y diámetro $(\mathrm{mm})$ en zona media y apical, se midieron con regla y calibrador Vernier respectivamente. El área superficial $\left(\mathrm{mm}^{2}\right)$ se ajustó por la semejanza a un cilindro recto por medio de la longitud y el promedio entre el diámetro de la base y medio de cada hoja. Igualmente se determinó el volumen $\left(\mathrm{cm}^{3}\right)$ que ocupa una bolsa de $500 \mathrm{~g}$ con cebollín. Se determinó la superficie específica del cebollín como la relación entre los valores promedio de área superficial y peso.

\section{Caracterización fisiológica}

El comportamiento fisiológico del cebollín para las condiciones establecidas, se caracterizó, a partir de la metodología propuesta por Parra y Hernández (2007), teniendo en cuenta la medición de las siguientes variables:

a) Intensidad respiratoria: se realizó utilizando el método de titulación de $\mathrm{CO}_{2}$ desprendido de la actividad respiratoria del producto. Se realizaron tres pruebas diarias para lo cual se usaron 100 g de cebollín en cada prueba, los cuales se colocaron en una cámara hermética de vidrio, la cual se conectó a un sistema de bombeo constante de aire provisto con una trampa de $\mathrm{CO}_{2} \mathrm{y}$ a un tubo de Petenkoffer, en el cual se colocaron $40 \mathrm{~mL}$ de hidróxido de bario $\left(\mathrm{Ba}(\mathrm{OH})_{2}{ }^{*} 2 \mathrm{H}_{2} \mathrm{O}\right)$ con normalidad de 0,1 , como reactivo de captura del $\mathrm{CO}_{2}$ generado en la respiración. El sistema se dejó funcionar por $20 \mathrm{~min}$, al cabo de los cuales se extrajo una muestra de $10 \mathrm{ml}$ del hidróxido de bario para titularla con ácido oxálico $\left(\mathrm{H}_{2} \mathrm{C}_{2} \mathrm{O}_{4}{ }^{*} 2\right.$
$\mathrm{H}_{2} \mathrm{O}$ ) con normalidad 0,1 . La intensidad respiratoria se determinó mediante la siguiente ecuación:

$R=\frac{\left(V_{b}-V_{m}\right) \times N \times 2}{w \times t}$

Donde:

$R$ : intensidad respiratoria (mg CO $\mathrm{kg}^{-1} \mathrm{~h}^{-1}$ )

$V_{b}$ : volumen del blanco, que es el volumen de ácido oxálico gastado al titular $10 \mathrm{~mL}$ de hidróxido de bario $\mathrm{Ba}(\mathrm{OH})_{2}$

$V_{m}$ : volumen de la muestra, es el volumen de ácido oxálico $\left(\mathrm{C}_{2} \mathrm{H}_{2} \mathrm{O}_{4}{ }^{*} 2 \mathrm{H}_{2} \mathrm{O}\right)$, gastado al titular 10 $\mathrm{mL}$ de hidróxido de bario $\mathrm{Ba}(\mathrm{OH})_{2}$ después de 20 min de respiración del producto $(\mathrm{mL})$

$N$ : normalidad del ácido oxálico $\left(\mathrm{C}_{2} \mathrm{H}_{2} \mathrm{O}_{4}{ }^{*} 2\right.$ $\left.\mathrm{H}_{2} \mathrm{O}\right): 0,1 \mathrm{~N}$

w: peso de la muestra, colocada en la cámara porta producto $(\mathrm{kg})$

$t$ : tiempo de circulación del aire a través del sistema $(\mathrm{h})$

b) Variación de peso: se determinó la pérdida de peso del cebollín colocando tres réplicas diarias por tratamiento, con una balanza de precisión; la variación se calculó en porcentaje (\%) por diferencia entre el peso inicial del almacenamiento y el peso del día de la medición.

\section{c) Determinación del contenido de humedad:} se empleó el método gravimétrico de la estufa. Por cada tratamiento se tomaron seis muestras diarias de producto, de aproximadamente $30 \mathrm{~g}$ y se llevaron a una estufa de convección forzada marca WTB Binder, a una temperatura de $105^{\circ} \mathrm{C}$ durante $7 \mathrm{~h}$, al cabo de las cuales se retiraron las muestras, se dispusieron en un frasco desecador durante 15 min y luego se pesaron en balanza Precisa XT220 ( $\pm 0,001$ g). La ecuación utilizada 
para determinar el contenido de humedad fue la siguiente:

$M(\%)=\frac{P_{\text {inicial }}-\text { Pfinal } \times 100}{P_{\text {inicial }}}$

Donde,

$M$ : contenido de humedad (\%)

$P_{\text {inicial: }}$ : peso inicial de la muestra, antes de colocarla en la estufa (g)

$P_{\text {final: }}$ peso final de la muestra, después de sacarla de la estufa $(\mathrm{g})$

\section{Análisis sensorial}

Para la evaluación sensorial de cebollín, el método de calificación aplicado fue un test con escala de cuatro puntos (donde: $4=$ muy buena calidad, $3=$ buena calidad, $2=$ calidad tolerable, $1=$ pobre calidad) para caracterizar: apariencia/turgencia $(A)$, color $(C)$, olor $(O)$, sabor $(T)$ y sanidad $(S)$, durante los ensayos de laboratorio, con relación al estado inicial del producto. Para facilitar el análisis cuantitativo de las calificaciones dadas por el panel sensorial, se determinó un índice de calidad total (ICT), que estimara porcentualmente la calidad general del producto; para ello, se establecieron coeficientes de ponderación para cada una de las características evaluadas, de acuerdo con las indicaciones dadas por los productores. El ICT se determinó entonces mediante la ecuación:

$I C T=(0,4 A+0,2 C+0,2 O+0,1 T+0,1 S) * 100 \%$

El ICT aceptable se estableció como mayor o igual a $70 \%$, considerado como el nivel de exigencia de calidad mínima que debe presentar el cebollín en el mercado. La evaluación se realizó cada día de prueba de laboratorio.

\section{Análisis estadístico}

Con los datos obtenidos durante la caracterización se calculó el coeficiente de variación, desviación estándar, mínimo y máximo. En la parte experimental se realizó un análisis de varianza (Anova). La significancia entre diferencias de medias fue determinada por la prueba de Tukey HSD a $P \leq 0,005$. Para el análisis de todos los datos fue usado el software Minitab bajo el entorno Windows.

\section{RESULTADOS Y DISCUSIÓN}

\section{Caracterización física}

Las características físicas de las hojas de cebollín fresco estudiado (tablas 1 y 2), presentaron coeficientes de variación de 24,97 y $23,97 \%$ para longitud y de 27,41 y 26,93\% para el diámetro medio, en la primera y segunda etapa respectivamente. Los coeficientes de variación para el peso fueron de $46,97 \%$ y $53,81 \%$ para la primera y segunda etapa respectivamente. Estos coeficientes de variación indican que hay cierta homogeneidad en las propiedades físicas del producto evaluadas en las dos fases de la investigación.

La longitud promedio del producto utilizado en la primera etapa fue de $33,6 \pm 8,33 \mathrm{~cm}$ y para la segunda etapa presentó una longitud promedio de 21,0 $\pm 5,03 \mathrm{~cm}$. Considerando la exportación y aceptación del cebollín en el mercado, la longitud recomendada debe estar entre 20 a $30 \mathrm{~cm}$ (Escalante y Soriano, 2008), lo cual lleva a pensar que la longitud no es un parámetro crítico en la comercialización del producto, o que en el país importador posiblemente se le realiza un manejo posterior, como reempacarlo en unidades más pequeñas "bunches" o "punches". La caracterización de la longitud denota la variación en las características físicas entre los materiales de exportación utilizados en las dos etapas, y es un primer supuesto de una posible variación en los resultados del estudio fisiológico entre las dos etapas que se establecieron. 
La superficie específica del cebollín se estableció en $4.834 \pm 1.200 \mathrm{~mm}^{2} \mathrm{~g}^{-1}$ y $5.526 \pm 900 \mathrm{~mm}^{2} \mathrm{~g}^{-1}$ para la primera y segunda etapa respectivamente, siendo estos valores aproximadamente cuatro veces mayores comparados con el valor reportado por Cantwell y Reid (1993) de $1.070 \mathrm{~mm}^{2}$ $\mathrm{g}^{-1}$ para el mismo producto. La cuantificación de esta característica física evidencia la susceptibilidad del cebollín a pérdidas considerables de agua de constitución y justifica la presencia de la película de agua que se observó en la superficie interna del empaque que contenía el producto durante toda la experimentación.

\section{Caracterización fisiológica}

La figura 1 indica que los valores medios de intensidad respiratoria para la condición refrigerada $\left(3^{\circ} \mathrm{C}\right)$ fueron de 293 y $94 \mathrm{mg} \mathrm{CO}_{2} \mathrm{~kg}^{-1} \mathrm{~h}^{-1}$ para los días 3 y 5 respectivamente y se estabilizó los días 7 al 13 con un valor promedio de $39 \mathrm{mg} \mathrm{CO}$ $\mathrm{kg}^{-1} \mathrm{~h}^{-1}$. En el día 15 presentó el menor valor de intensidad respiratoria correspondiente a 20,5 mg $\mathrm{CO}_{2} \mathrm{~kg}^{-1} \mathrm{~h}^{-1}$, este día se presentaron síntomas de malos olores (característico a alcohol) y por la regular apariencia visual se culminaron las pruebas de intensidad respiratoria, pero se continuó con el análisis sensorial. Hasta el día 11 para la condición refrigerada, las cualidades del producto fueron aceptables según los criterios establecidos. Sin embargo, hay un ligero aumento en la intensidad respiratoria el día 13, posiblemente ocasionado en gran parte por las condiciones de alta humedad que presentaba el conjunto producto-empaque. Dentro del seguimiento visual se puede inferir que para este día la cantidad de agua producida por el cebollín y retenida por el empaque fue tal que ya no podía retener más agua y se formó un ambiente húmedo, lo que llevó a un deterioro generalizado del producto que se hizo crítico el día 15.

La intensidad respiratoria del producto almacenado a condición ambiente $\left(18^{\circ} \mathrm{C}\right)$ presenta un intervalo de confianza amplio en el día 3 , el cual se asoció a una alta heterogeneidad en la inten- sidad respiratoria del producto (dispuesto en el empaque-embalaje); el valor obtenido para este día fue de $318,43 \mathrm{mg} \mathrm{CO}_{2} \mathrm{~kg}^{-1} \mathrm{~h}^{-1}$, reduciéndose a $204,31 \mathrm{mg} \mathrm{CO}_{2} \mathrm{~kg}^{-1} \mathrm{~h}^{-1}$ el día 5 , hasta cuando conservó su calidad comercial. En el día 7 de almacenamiento las características en la apariencia del producto no justificaron la continuación de la pruebas para el producto almacenado a $18^{\circ} \mathrm{C}$. Los valores de intensidad respiratoria encontrados en la presente investigación están alejados de los encontrados por Sanabria (2004) y Ángel (2004), debido fundamentalmente a que los estudios realizados por estos dos autores no presentan una tendencia respiratoria definida, causada posiblemente por deficiencias en las condiciones experimentales utilizadas. Sin embargo, Sanabria (2004) reporta que el cebollín almacenado a $18^{\circ} \mathrm{C}$ mantuvo su calidad durante 5 días, lo cual concuerda con lo reportado por Medina (1988) para cebolla junca y con los resultados encontrados en la presente investigación.

En general, para la primera etapa (figura 1) las curvas de intensidad respiratoria muestran valores altos en los primeros días de almacenamiento y en los días posteriores la curva tiene una tendencia descendente, mostrando el comportamiento típico de un producto no climatérico, tendencia similar a la encontrada por Medina (1988) para cebolla junca. Para el cebollín refrigerado la intensidad respiratoria se estabiliza del día 7 al 13. Estadísticamente no hay diferencia en los niveles de intensidad respiratoria del cebollín en los días 3 y 5 de almacenamiento entre las dos pruebas. Se deduce que la temperatura de $3^{\circ} \mathrm{C}$ comparada con la de $18^{\circ} \mathrm{C}$ permitió que el cebollín conservará su calidad en almacenamiento por un tiempo mayor, debido a que la baja temperatura retarda directamente la velocidad con la que se desarrollan los procesos fisiológicos y biológicos que conllevan a la pérdida de calidad de estos productos (Chomchalow, 2002).

La intensidad respiratoria de la segunda etapa (figura 2) presentó un comportamiento generalizado en los cuatro tratamientos durante los $8 \mathrm{~d}$ 


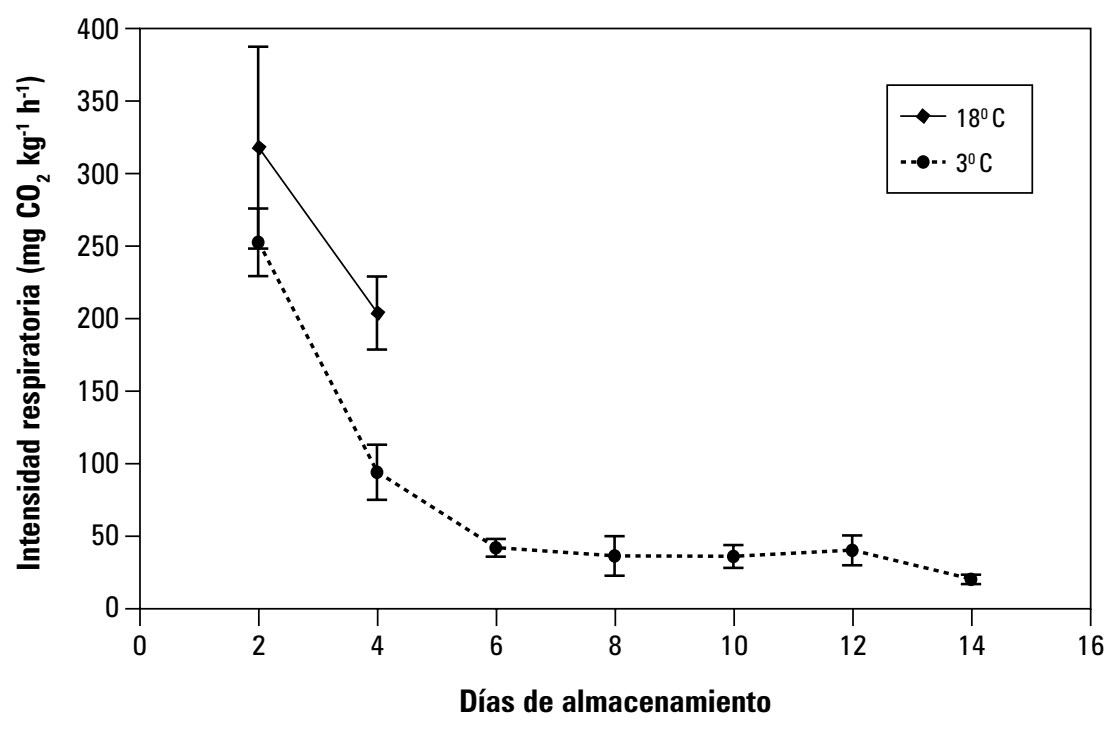

Figura 1. Intensidad respiratoria de cebollín a $3^{\circ} \mathrm{C}$ y $18^{\circ} \mathrm{C}$. Los datos representan un intervalo de confianza del $95 \%$ para la media de tres réplicas de la muestra.

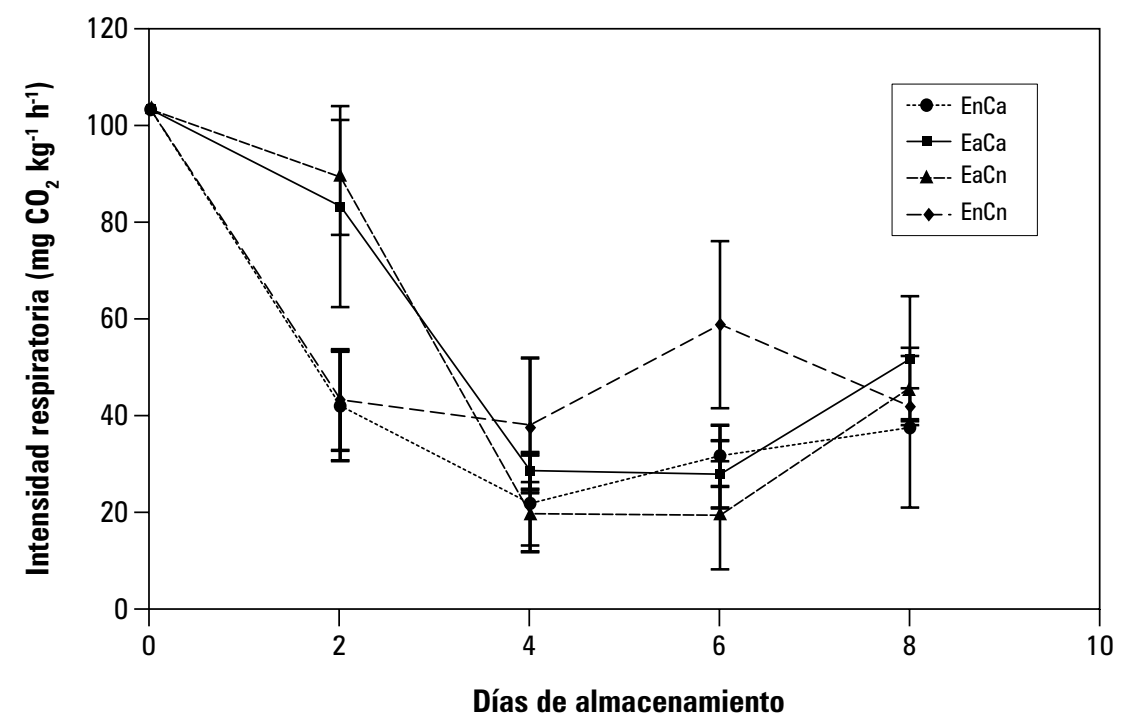

Figura 2. Intensidad respiratoria de cebollín en los tratamientos EaCa (empaque actual caja actual), EaCn (empaque actual caja nueva), EnCa (empaque nuevo caja actual), EnCn (empaque nuevo caja nueva). Los datos representan un intervalo de confianza del $\mathbf{9 5 \%}$ para la media de tres réplicas de la muestra.

de almacenamiento, siendo mayores los valores al inicio del almacenamiento $\left(103 \mathrm{mg} \mathrm{CO} \mathrm{kg}^{-1}\right.$ $\left.\mathrm{h}^{-1}\right)$. En el día 2 de almacenamiento, los tratamientos con empaque propuesto disminuyeron su actividad en mayor proporción que los del empaque actual. En el día 6 de almacenamiento se presentó, con excepción del tratamiento de empaque y embalaje propuesto que generó un pico en su intensidad respiratoria de $59 \mathrm{mg} \mathrm{CO}_{2}$ $\mathrm{kg}^{-1} \mathrm{~h}^{-1}$, un descenso en los demás tratamientos hasta un valor promedio de $26 \mathrm{mg} \mathrm{CO}_{2} \mathrm{~kg}^{-1} \mathrm{~h}^{-1}$. Al final del almacenamiento (día 8) se hizo evi- 
dente el comportamiento mostrado en el día 2, en el cual los tratamientos con empaque actual mostraron los valores más altos de $45 \mathrm{mg} \mathrm{CO}_{2}$ $\mathrm{kg}^{-1} \mathrm{~h}^{-1}, \mathrm{y}$ los de empaque propuesto, valores de $24 \mathrm{mg} \mathrm{CO} \mathrm{kg}^{-1} \mathrm{~h}^{-1}$.

El nivel de significancia estadística entre los valores medios para cada tratamiento para un intervalo de confianza del $95 \%$ de la media, advierten la poca relevancia de empacar el cebollín con películas de polietileno de baja densidad con un nivel de perforación mayor (1,38\%) al actual (1\%). Este resultado se atribuye a la forma como se dispone el producto con empaque plástico dentro del embalaje para la exportación, disposición que no garantiza las áreas de intercambio gaseoso (área perforada). Los empaques con 500 $\mathrm{g}$ de producto cada uno se disponen verticalmente y muy juntos entre sí dentro del embalaje, con el fin de tener una mayor cantidad de producto y de esta forma reducir los costos de envió. No obstante, se resalta que el tratamiento empaque propuesto-embalaje actual (EnCa; figura 2) presentó una tendencia en la actividad respiratoria con valores medios menores a los otros tratamientos.
En la primera etapa, el contenido de humedad del conjunto cebollín-empaque no varió significativamente en el tiempo de almacenamiento de cada uno de los tratamientos, ni entre los dos tratamientos comparados hasta el día 5 (figura 3); el valor promedio de contenido de humedad durante la experiencia se estableció en $91,5 \%$. El contenido de humedad en la segunda etapa (figura 4), para el conjunto cebollín-empaque, varió desde un valor inicial cercano al $91 \%$ hasta un valor cercano a $82 \%$ en el día 8 de almacenamiento $\left(\right.$ a $3^{\circ} \mathrm{C}$ ) para todos los tratamientos, siendo ligeramente menor en los tratamientos con el embalaje actual (EaCa y EnCa), debido a la barrera física al paso del agua que este provee. La mayor pérdida en los embalajes con perforación (EaCn y EaCn) es ocasionada por el intercambio entre el producto y el aire del cuarto de almacenamiento y el del espacio libre dentro del empaque; por tanto, el embalaje propuesto no es una buena opción para el almacenamiento de cebollín bajo las condiciones de almacenamiento en que se realizaron las evaluaciones.

Las pérdidas de peso del conjunto productoempaque a lo largo del experimento, fueron

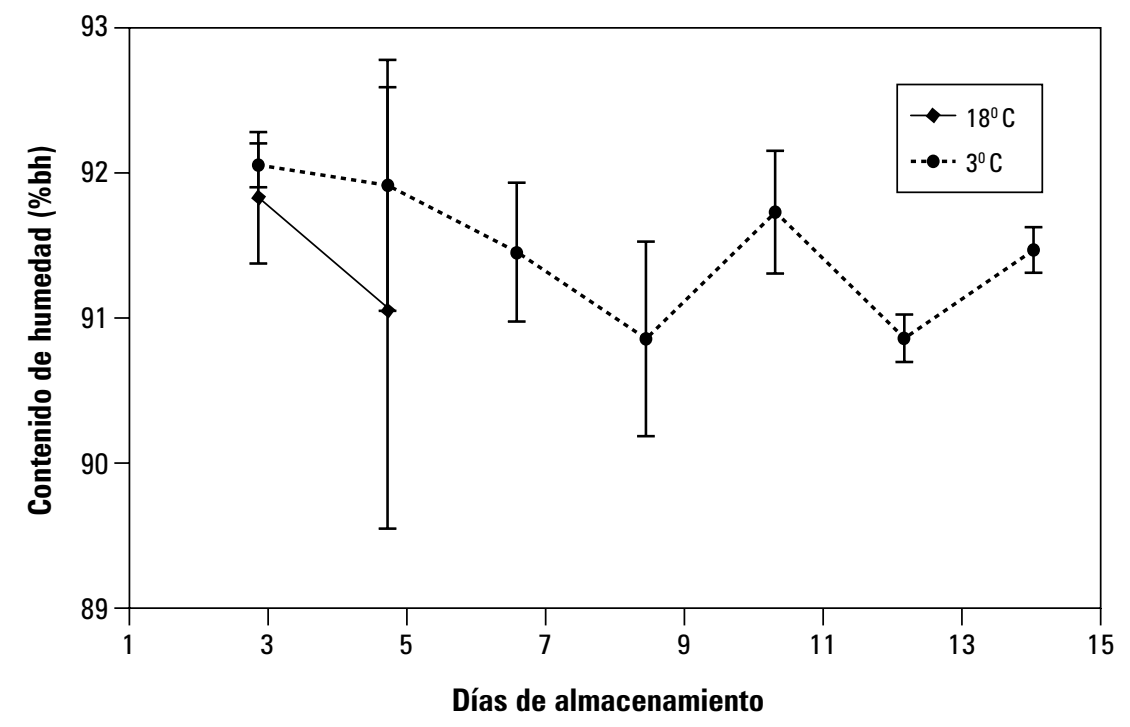

Figura 3. Contenido de humedad para cebollín a $3^{\circ} \mathrm{C}$ y $18^{\circ} \mathrm{C}$. Los datos representan un intervalo de confianza del 95\% para la media de cinco réplicas de la muestra. 


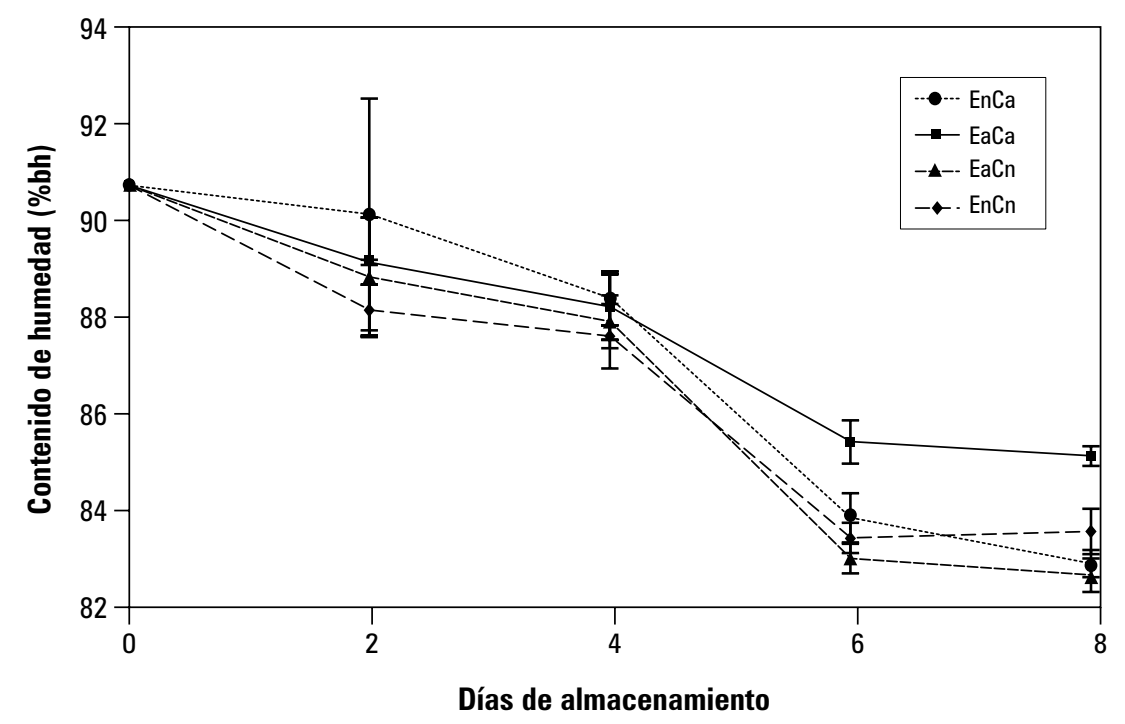

Figura 4. Contenido de humedad para los tratamientos EaCa (empaque actual caja actual), EaCn (empaque actual caja nueva), EnCa (empaque nuevo caja actual), EnCn (empaque nuevo caja nueva). Los datos representan un intervalo de confianza del $\mathbf{9 5 \%}$ para la media de tres réplicas de la muestra.

menores al $4 \%$ en todos los tratamientos de la segunda etapa, registrándose los mayores valores en el embalaje con $9 \%$ de perforación. Para $8 \mathrm{~d}$ de almacenamiento, se considera un valor relevante por la implicación en las pérdidas de turgencia del producto. Se debe resaltar que el producto de la primera etapa tuvo pérdidas por traspiración inferiores al 3\%, durante $15 \mathrm{~d}$ de almacenamiento. Estos porcentajes de pérdida de agua pueden estar relacionados con una alta relación superficie/volumen y un gran número de estomas en los tejidos verdes de la hoja, lo que resulta en altas tasas de pérdida de agua por transpiración y se traduce en pérdidas de peso y de calidad visual (Cantwell y Reid, 1993; Sandhya, 2009).

Ángel (2004) estableció valores de pérdida de peso para unidades experimentales no dispuestas en embalajes para las condiciones evaluadas de 5 a $7 \%$ al final del almacenamiento (15 d). Esta mayor pérdida de peso comparada con los datos que se reportan en este documento, puede ser debida al déficit de presión de vapor entre el aire del cuarto refrigerado y las unidades experimentales, lo cual facilitó la evaporación del agua del empaque. En albahaca, bajo las condiciones estudiadas por Camargo (2008) se encontraron pérdidas de 0,9\% para la condición más crítica. Según lo reportado por Mir y Beaudry (2004), la pérdida de agua a través de películas plásticas no supera el $5 \%$, lo cual concuerda con los resultados obtenidos en la presente investigación (pérdidas de peso inferiores a 4\%). Por ende, la película de empaque evita altas tasas de pérdida de agua por transpiración.

Teniendo en cuenta los resultados de contenido de humedad del cebollín con los valores de pérdida de peso, se deduce que el empaque actual desempeña un papel importante para el mantenimiento del contenido de humedad y ocurre poca variación de peso por transpiración del conjunto producto-empaque, debido posiblemente a la AM y saturada que se crea en el espacio entre el empaque y el producto, lo cual puede inhibir los procesos fisiológicos y biológicos de manera benéfica. Resultados similares han sido presentados para hierbas frescas dispuestas en empaques de polietileno de baja densidad por Ángel (2004), Sanabria (2004) y Bedoya (2005). 
Se establece entonces que las combinaciones: bolsa de polietileno de baja densidad con un nivel de perforación de 1,38\% y la caja telescópica de exportación (empaque nuevo propuesto embalaje actual, respectivamente), ayudan en la conservación del cebollín a una temperatura de $3^{\circ} \mathrm{C}$, debido a que se favorece la retención de agua y minimiza la pérdida de peso. El producto almacenado a temperatura de $18^{\circ} \mathrm{C}$ bajo las condiciones de estudio sufrió una rápida deterioración del producto, lo cual ratifica la necesidad del manejo del cebollín bajo condiciones refrigeradas.

No obstante se debe considerar técnica y económicamente otros tipos de películas de empaque con una adecuada permeabilidad al vapor de agua, $\mathrm{CO}_{2} \mathrm{y} \mathrm{O}_{2}$ o el purgado con estos gases al momento de empacar el producto, que permita mejorar el comportamiento poscosecha del cebollín en bolsas de polietileno de baja densidad perforadas.

\section{Análisis sensorial}

De acuerdo con la metodología establecida en la primera etapa, se determinó el ICT para la condición ambiente de $79 \%$ a los 5 d de almacenamiento y de $73 \%$ para la condición refrigerada el día 11 de almacenamiento, aunque en los días posteriores el material a condición ambiente se descompuso rápidamente arrojando un ICT de 33\%, mientras que el expuesto a condición refrigerada permitió mantenerse con una calidad según el ICT del 68\% el día 13 y 34\% el día 15. Para la segunda etapa, la evaluación sensorial no evidenció diferencias marcadas entre los tratamientos; en general se alcanzó un ICT de $75 \%$ a los $6 \mathrm{~d}$ de almacenamiento, y a los $8 \mathrm{~d}$ un ICT de $60 \%$ para el tratamiento empaque propuesto-embalaje actual (EnCa) y menores a $55 \%$ para los demás tratamientos. En general para toda la investigación, las características de sanidad (siendo este un parámetro importante para la exportación a los Estados Unidos) fueron excelentes debido a un correcto manejo agronómico del cultivo en campo. Durante los últimos días de almacenamiento en todos los tratamientos, se evidenciaron olores desagradables y un amarillamiento generalizado de las hojas de cebollín, siendo las hojas más pequeñas o de mayor superficie específica, las primeras en presentar este síntoma. La apariencia final del conjunto producto-empaque fue desagradable.

\section{CONCLUSIONES}

La calidad del cebollín se conserva adecuadamente mediante el manejo del producto a bajas temperaturas $\left(3^{\circ} \mathrm{C}\right)$ durante toda su comercialización, ya que esta disminuye directamente la velocidad con la que se desarrollan los procesos fisiológicos y biológicos que conducen a la pérdida de calidad de este producto. El comportamiento fisiológico del cebollín depende de la temperatura de almacenamiento y del empaque con el que se disponga.

Tabla 1. Características físicas de las hojas de cebollín para los parámetros longitud, diámetro (base y medio), peso y área superficial, para la primera etapa.

\begin{tabular}{|c|c|c|c|c|c|}
\hline \multirow{2}{*}{ Parámetro } & \multirow{2}{*}{ Longitud (cm) } & \multicolumn{2}{|c|}{ Diámetro (cm) } & \multirow{2}{*}{ Peso (g) } & \multirow{2}{*}{$\begin{array}{c}\text { Área superficial } \\
\qquad\left(\mathrm{mm}^{2}\right)\end{array}$} \\
\hline & & base & medio & & \\
\hline Promedio & 33,40 & 3,16 & 2,61 & 0,648 & $3.131,92$ \\
\hline Desviación estándar & 8,330 & 0,775 & 0,715 & 0,304 & $1.323,695$ \\
\hline Coeficiente de variación (\%) & 24,97 & 24,49 & 27,41 & 46,97 & 42,26 \\
\hline Máximo & 49,90 & 5,00 & 4,50 & 1,490 & 7446,36 \\
\hline Mínimo & 17,50 & 1,60 & 1,10 & 0,199 & 933,05 \\
\hline
\end{tabular}


Tabla 2. Características físicas de las hojas de cebollín para los parámetros longitud, diámetro (base y medio), peso y área superficial, para la segunda etapa.

\begin{tabular}{|l|c|c|c|c|c|}
\hline \multirow{2}{*}{ Parámetro } & \multirow{2}{*}{ Longitud $(\mathrm{cm})$} & \multicolumn{2}{c|}{ Diámetro $(\mathrm{cm})$} & \multirow{2}{*}{ Peso $(\mathrm{g})$} & $\begin{array}{c}\text { Área superficial } \\
\left(\mathrm{mm}^{2}\right)\end{array}$ \\
\cline { 3 - 6 } & & base & medio & & $1.699,39$ \\
\hline Promedio & 21,00 & 2,75 & 2,25 & 0,308 & 731,970 \\
\hline Desviación estándar & 5,030 & 0,647 & 0,606 & 0,165 & 43,07 \\
\hline Coeficiente de variación (\%) & 23,97 & 23,50 & 26,93 & 53,81 & $3.988,80$ \\
\hline Máximo & 33,00 & 4,90 & 4,45 & 0,876 & 627,69 \\
\hline Mínimo & 11,00 & 1,70 & 1,35 & 0,094 & 6 \\
\hline
\end{tabular}

Las bolsas perforadas de polietileno de baja densidad utilizadas actualmente colaboran para la retención de humedad del producto. Se considera deseable un porcentaje de área de perforación del área total de la bolsa cercano a 1,38\%, perforaciones de diámetro de $4 \mathrm{~mm}$, con buena uniformidad en su distribución en el área de la película para mejorar la eficacia de ésta. Sin embargo, los resultados presentados en esta investigación y considerando la forma de empaque actual con la que se comercializa el producto, sugieren que el uso de otro tipo de películas con diferente permeabilidad a la actual, podría no aumentar la prolongación de la vida poscosecha del cebollín.

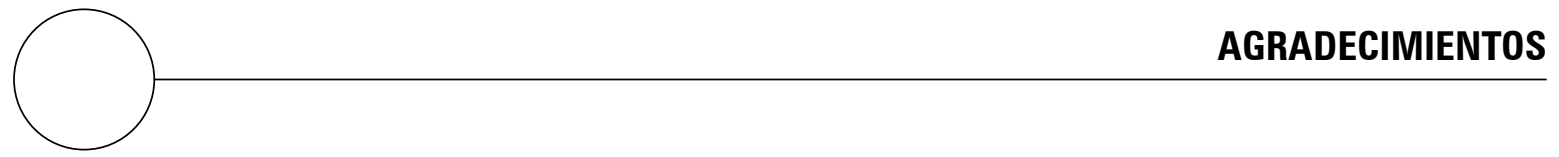

Los autores agradecen a la Universidad Nacional de Colombia, sede Bogotá y a sus laboratorios de Poscosecha de Productos Agrícolas del Departamento de Ingeniería Civil y Agrícola, donde se realizó la investigación, contando con la financiación del Macroproyecto "Corredor Tecnológico Agroindustrial", subproyecto de empaques y embalajes.

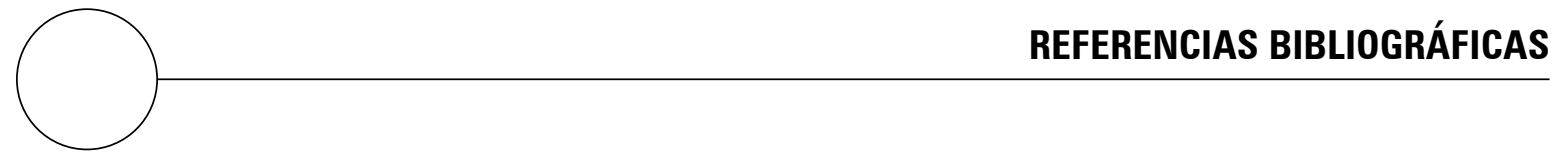

Abello, J., J. Clavijo y P. Barreno. 2006. Estudio preliminar de algunos descriptores fisiológicos en cinco hierbas aromáticas. pp. 13-15. En: Memorias Curso de Extensión: Últimas tendencias en hierbas aromáticas culinarias para exportación en fresco. Facultad de Agronomía, Universidad Nacional de Colombia, Bogotá.

Ángel, D. 2004. Efecto del empaque de polietileno en la calidad del cebollín (Allium schoenoprasum) en almacenamiento refrigerado. Trabajo de grado. Facultad de Ingeniería, Universidad Nacional de Colombia, Bogotá.
Barreño, P. 2005. Cebollín (Allium schoenoprasum). Memorias de hierbas para exportación en fresco. Facultad de Agronomía, Universidad Nacional de Colombia, Bogotá.

Barreño, P. 2006. Hierbas aromáticas culinarias para exportación en fresco manejo agronómico, producción y costos. pp. 65-72. En: Memorias Curso de Extensión: Últimas tendencias en hierbas aromáticas culinarias para exportación en fresco. Facultad de Agronomía, Universidad Nacional de Colombia, Bogotá. 
Barreño, P. y J. Clavijo. 2006. Hierbas aromáticas culinarias para exportación en fresco. pp. 7-9. Memorias Curso de Extensión: Últimas tendencias en hierbas aromáticas culinarias para exportación en fresco. Facultad de Agronomía, Universidad Nacional de Colombia, Bogotá.

Bedoya, L. 2005. Caracterización física y fisiológica poscosecha de dos hierbas aromáticas condimentarias: Menta (Mentha spicata L.) y Mejorana (Origanum majorana L.) a tres temperaturas y dos condiciones de almacenamiento. Trabajo de grado. Facultad de Ingeniería, Universidad Nacional de Colombia, Bogotá.

Bernal, D.A., L.C. Morales, G. Fischer, J. Cuervo y S. Magnitski. 2008. Caracterización de las deficiencias de macronutrientes en plantas de cebollín (Allium schoenoprasum L.). Rev. Colomb. Cienc. Hortíc. 2(2), 192-204.

Camargo, N. 2008. Conservación de la albahaca (Ocimun basilicum) fresca en refrigeración, empacada en películas plásticas con atmósferas modificadas. Trabajo final. Especialización en Ciencia y Tecnología de Alimentos, Universidad Nacional de Colombia, Bogotá.

Cantwell, M.I. y M.S. Reid. 1993. Postharvest physiology and handling of fresh culinary herbs. J. Herbs Spices Medicinal Plants 1(3), 93-127.

Chomchalow, N. 2002. Production of herbs in Asia: An overview. AU J.T. 6(2), 95-108.

Delahaut, K.A. y A.C. Newenhouse. 2003. Growing onions, garlic, leeks, and other Allium in Wisconsin: A guide for fresh-market growers. Cooperative Extension, University of Wisconsin, Madison, WI.

Escalante, J. y J. Soriano. 2008. Determinación de las fases de desarrollo fenológico de cebollín (Allium schoenoprasum) mediante la escala bbch y la técnica grados día de crecimiento (GDC). Trabajo de grado. Facultad de Agronomía, Universidad Nacional de Colombia, Bogotá.

Imahori, Y., Y. Suzuki, K. Uemura, I. Kishioka, H. Fujimara, Y. Ueda y K. Chachin. 2004. Physiological and quality responses of Chinese chive leaves to low oxygen atmosphere. Postharvest Biol. Technol. 31, 295-303.

Imahori, Y., Y. Suzuki, M. Kawagishi, M. Ishimaru, Y. Ueda y K. Chachin. 2007. Physiological responses and quality attibutes of Chinese Chive leaves exposed to $\mathrm{CO}_{2}$-enriched atmospheres. Postharvest Biol. Technol. 46, 160-166.

Medina, R. 1988. Evaluación de pérdidas en la poscosecha de la cebolla junca. Trabajo de grado. Facultad de Ingeniería, Universidad Nacional de Colombia, Bogotá.

Mir, N. y R. Beaudry. 2004. Modified Atmosphere packaging. Michigan State University, East Lansing, MI.

Parra, A. 2007. Técnicas de almacenamiento y conservación de frutas y hortalizas frescas. Unidad de Publicaciones de la Facultad de Ingeniería, Universidad Nacional de Colombia, Bogotá.

Parra, A. y J.E. Hernández. 2007. Fisiología de poscosecha de frutas y hortalizas. Cuarta edición. Taller de Publicaciones. Facultad de Ingeniería, Universidad Nacional de Colombia, Bogotá.

Sanabria, B. 2004. Caracterización física y fisiológica poscosecha de dos hierbas aromáticas condimentarias: cebollín (Allium schoenoprasum) y orégano (Origanum vulgare L.) a tres temperaturas, almacenado con y sin empaque. Trabajo de grado. Facultad de Ingeniería, Universidad Nacional de Colombia, Bogotá.

Sandhya. 2009. Modified atmosphere packaging of fresh produce: Current status and future needs. LWTFood Sci. Technol. 43(3), 381-392.

Smyth, A.B., J. Song y A.C. Cameron. 1998. Modified atmosphere packaged cut iceberg lettuce: effect of temperature and $\mathrm{O}_{2}$ partial pressure on respiration and quality. J. Agric. Food Chem. 46, 45564562 .

Yahia, E. 2009. Modified and controlled atmospheres for the storage, transportation, and packaging of horticultural commodities. CRC Press, Taylor \& Francis, Boca Raton, FL. 